\title{
Segmental Transarterial Embolization in a Translational Rat Model of Hepatocellular Carcinoma
}

\author{
Terence P.F. Gade, MD, PhD, Stephen J. Hunt, MD, PhD, Neil Harrison, MD, Gregory J. \\ Nadolski, MD, Charles Weber, MD, Stephen Pickup, PhD, Emma E. Furth, MD, Mitchell D. \\ Schnall, MD, PhD, Michael C. Soulen, MD, and M. Celeste Simon, PhD \\ Penn Image-Guided Interventions Laboratory (T.P.F.G., S.J.H., N.H., G.J.N., C.W.), University of \\ Pennsylvania; and Departments of Radiology (T.P.F.G., S.J.H., G.J.N., C.W., S.P., M.D.S., \\ M.C.So.) and Pathology and Laboratory Medicine (E.E.F.), Abramson Family Cancer Research \\ Institute (M.C.Si.), and Howard Hughes Medical Institute (M.C.Si.), Perelman School of Medicine \\ at the University of Pennsylvania (N.H.), 421 Curie Boulevard, 438 BRB II/III, Philadelphia, PA \\ $19104-6160$
}

\begin{abstract}
Purpose-To develop a clinically relevant, minimally invasive technique for transarterial embolization in a translational rat model of hepatocellular carcinoma (HCC).

Materials and Methods-Oral diethylnitrosamine was administered to 53 male Wistar rats ad libitum for 12 weeks. Tumor induction was monitored using magnetic resonance imaging. Minimally invasive lobar or segmental transarterial embolization was performed through a left common carotid artery approach. Necropsy was performed to evaluate periprocedural mortality. Histologic analysis of tumors that received embolization was performed to assess percent tumor necrosis.
\end{abstract}

\begin{abstract}
Results-Severe cirrhosis and autochthonous HCCs were characterized in a cohort of rats composed of two groups of rats identically treated with diethylnitrosamine with median survival times of 101 days and 105 days ( $n=10$ /group). A second cohort was used to develop minimally invasive transarterial embolization of HCCs $(n=10)$. In a third cohort, lobar embolization was successfully performed in 9 of 10 rats and demonstrated a high rate of periprocedural mortality (n $=5$ ). Necropsy performed for periprocedural mortality after lobar embolization demonstrated extensive tissue necrosis within the liver $(\mathrm{n}=3)$ and lungs $(\mathrm{n}=2)$, indicating nontarget embolization as the likely cause of mortality. In a fourth cohort of rats, a segmental embolization technique was successfully applied in 10 of 13 rats. Segmental embolization resulted in a reduction in periprocedural mortality $(P=.06)$ relative to selective embolization and a $19 \%$ increase in average tumor necrosis $(P=.04)$.
\end{abstract}

Conclusions-Minimally invasive, segmental embolization mimicking the currently applied clinical approach is feasible in a translational rat model of HCC and offers the critical advantage of reduced nontarget embolization relative to lobar embolization.

\footnotetext{
Address correspondence to M.C.Si.; celeste2@mail.med.upenn.edu.

None of the authors have identified a conflict of interest.
} 
Advances in tumor biology have led to the identification of molecular and cell-based therapies that may augment the therapeutic effect of transarterial embolization or transarterial chemoembolization, or both $(1,2)$. Translation of these therapies requires an animal model that recapitulates the biology of the human disease, while allowing the use of a minimally invasive embolization technique that reflects current clinical protocols. Animal models of transarterial chemoembolization reported in the literature underscore the challenge of developing a model with these attributes.

Although the ideal animal model would encompass the varied and complex biology embodied by hepatocellular carcinoma (HCC), two features are of particular relevance with respect to transarterial embolization and transarterial chemoembolization. First, most human HCCs develop in the background of cirrhosis, which has been shown to play a role in pathogenesis $(3,4)$. The resultant hepatocellular dysfunction influences the approach to transarterial embolization or transarterial chemoembolization, or both, given the associated diminution in hepatic reserve and potential for vascular abnormalities. This dysfunction emphasizes the importance of superselective over lobar embolization to preserve functional hepatic parenchyma and to enhance therapeutic efficacy $(5,6)$. Second, the dependence of HCCs on the arterial blood supply develops gradually through the growth of abnormal intranodular arteries, a feature that has been shown to occur in autochthonous tumor models and not in spontaneous tumor models $(7,8)$. Chemically induced, autochthonous, small and large animal models of HCC encompassing both of these features have been described (911); however, these models have not been used for studies of transarterial embolization and transarterial chemoembolization preclinical application. Small animal models, including mice and rats, offer advantages including ease of handling, low procurement and maintenance costs, less extensive anesthesia and monitoring protocols during the procedure, and the potential for high throughput and established methods for biologic and genetic manipulation. The size of the vasculature in these animals has precluded the use of the clinically applied technique of minimally invasive, superselective embolization. For example, the diameter of the proper hepatic artery in Wistar rats weighing 250-300 g measures only $0.2-0.5 \mathrm{~mm}$ (12). None of the currently reported small animal models of transarterial embolization or transarterial chemoembolization, or both, describe selective embolization beyond the lobar hepatic artery.

In the present study, we describe and characterize the efficacy of minimally invasive, segmental transarterial embolization in a translational rat model of HCC. Cirrhosis and subsequent autochthonous HCCs were induced through the ad libitum oral administration of the hepatocarcinogen diethylnitrosamine (DEN). Embolization of either lobar or segmental hepatic arteries was achieved through a left common carotid artery (LCCA) approach with primary closure of the arteriotomy and sustained patency of the LCCA. This technique enables the opportunity for studies of transarterial embolization or transarterial chemoembolization, or both, in a clinically relevant model of HCC. 


\section{MATERIALS AND METHODS}

\section{Animal Model}

Animal studies were conducted according to institutionally approved protocols for the safe and humane treatment of animals. Male Wistar rats 4 weeks old (Charles River Laboratories International, Inc, Wilmington, Massachusetts) were acclimated to the animal facility for 2 weeks. Autochthonous HCCs were induced in rats $(n=53)$ using ad libitum oral intake of $0.01 \%$ DEN (Sigma-Aldrich, St. Louis, Missouri) for 12 weeks, and the rats were divided among four cohorts (Table) (13). The water bottles were changed twice per week with freshly prepared $0.01 \%$ DEN. Animals were closely monitored over the course of therapy and weighed twice weekly. Single primary operator cohorts of 10 animals exposed to DEN (groups 1 and 2), baseline growth (eg, weight gain), and median survival were characterized for untreated (no embolization was performed) rats. Weight gain from these baseline groups was compared with published data for male Wistar rats not receiving DEN (14). Animals demonstrating weight loss $>20 \mathrm{~g}$ or diminished grooming capability were euthanized using inhaled carbon dioxide (20\%). Necropsy was performed on euthanized animals; selected animals were submitted for formal necropsy performed by a veterinary pathologist.

\section{MR Imaging}

Magnetic resonance (MR) imaging was performed 1 day after the cessation of DEN treatment and weekly thereafter until the time of experiment or expiration. MR imaging was performed using a Varian 4.7-tesla 40-cm horizontal bore MR spectrometer with a 25 gauss/cm gradient tube interfaced to a Varian DirectDrive console (Agilent Technologies, Santa Clara, California). Animals were induced and maintained under inhalational anesthesia using isoflurane (2\%) and positioned within a high-pass birdcage resonator that was tuned and matched to $200 \mathrm{MHz}$. Respiratory gated, T2-weighted images were acquired in the axial plane with $55 \mathrm{~mm}$ field of view, $128 \times 128$ matrix size, $430 \mu \mathrm{m}$ in-plane resolution, $2 \mathrm{~mm}$ slice thickness, 0 interslice gap, 4 signal averages, $6.3 \mathrm{~ms}$ repetition time, and $2.98 \mathrm{~ms}$ echo time. Image analysis, including measurements of tumor diameters, was performed using Agilent VnmrJ 2.3A software (Agilent Technologies).

\section{Transarterial Embolization}

Animals bearing tumors measuring a minimum of $0.5 \mathrm{~cm}$ in transverse diameter on MR imaging were selected to undergo embolization. Experiments were performed using an AngioStar Plus Imaging System (Siemens, Malvern, Pennsylvania). Nonsurvival experiments were performed using a single cohort of 10 rats for the development of minimally invasive hepatic transarterial embolization with cannulation of the common hepatic artery (CHA) based on a previously described LCCA approach (15). Following this initial developmental cohort, a group of 10 animals underwent lobar hepatic embolization, whereas another cohort of 13 animals underwent segmental embolization (Table).

Transarterial embolization was performed under $2 \%$ isoflurane inhaled using a nose cone with body temperature maintained at $37^{\circ} \mathrm{C}$ using a circulating water blanket and monitored using a rectal temperature probe. Blunt left neck dissection was performed through a 4-cmlong skin incision to expose the LCCA. Using a stereomicroscope (Leica Microsystems AG, 
Heerbrugg, Switzerland) under $30 \times$ magnification, the carotid sheath was dissected, and the LCCA was isolated from the left vagus nerve to expose a $2-\mathrm{cm}$ segment of vessel. Vessel loops of 5-0 silk suture were placed at proximal and distal most segments of the LCCA to facilitate hemostasis; 7-mm vascular clamps (jaw dimensions, $3.5 \mathrm{~mm} \times 1 \mathrm{~mm}$; Fine Science Tools, Foster City, California) were then positioned adjacent to the 5-0 sutures at the proximal and distal most segments of the LCCA. An arteriotomy was created in the center of the exposed segment using a 26-gauge Angiocath (outer diameter, $0.46 \mathrm{~mm}$; BD Biosciences, San Jose, California). An 8-0 suture (Fine Science Tools) was placed in the anterior lip of the arteriotomy to facilitate introduction of the catheter and closure of the arteriotomy. The proximal vascular clamp was removed, and hemostasis was maintained through retraction of the proximal 5-0 suture. A 1.5-F (outer diameter, $0.5 \mathrm{~mm}$ ), preshaped ( $\mathrm{J}$ or $90^{\circ}$ ) microcatheter (Excelsior SL-10 Microcatheter, Stryker, Kalamazoo, Michigan) custom modified to $30 \mathrm{~cm}$ was introduced into the LCCA with the aid of a coaxially inserted 0.010-inch guide wire (Transend guide wire; Stryker). On fluoroscopic confirmation of guide wire positioning within the descending aorta, the catheter was advanced over the wire into the abdominal aorta. The guide wire was withdrawn, and the celiac artery (T12-L1 interspace) was catheterized. A celiac arteriogram was obtained to delineate the relevant anatomy using iopamidol contrast agent (Isovue 370, $0.3 \mathrm{~mL}$; Bracco Diagnostics, Inc, Monroe Township, New Jersey). Transarterial embolization was subsequently performed using a lobar or segmental technique with hand injection of $0.1 \mathrm{~mL}$ of sedimented 40-120 $\mu \mathrm{m}$ Embospheres (Merit Medical Systems, South Jordan, Utah) diluted in $0.1 \mathrm{~mL}$ Isovue 370 using a $1-\mathrm{mL}$ polycarbonate syringe. The catheter was subsequently flushed with 0.1 $\mathrm{mL}$ of saline given catheter dead volume of approximately $0.06 \mathrm{~mL}$. Rats treated with transarterial embolization received 3 days of analgesia after the procedure with daily administration of subcutaneous meloxicam $(2 \mu \mathrm{g} / \mathrm{kg})$. Treated animals were closely monitored and weighed daily for 3 days after treatment. Rats were monitored until they met the criteria for euthanasia described earlier or until they expired, at which time necropsy was performed. The percentage necrosis was determined for each HCC on hematoxylin-eosin (H\&E)-stained tumor sections in the untreated control cohorts and for targeted tumors in the cohorts with embolization by a gastrointestinal pathologist (E.E.F.) with extensive experience in evaluating $\mathrm{HCC}$ specimens after transarterial embolization and transarterial chemoembolization.

\section{Tissue Preparation and Histology}

After euthanasia or expiration, tissues were harvested, fixed in $4 \%$ paraformaldehyde, and dehydrated in ethanol. Dehydrated tissues were embedded in paraffin and sectioned. H\&E and Masson trichrome staining of tissue sections was performed using standard protocols. Embedded tissues were deparaffinized in an oven at $55^{\circ} \mathrm{C}$ followed by application of xylene. For H\&E staining, tissues were rehydrated in ethanol, stained with H\&E, mounted using Permount (Thermo Fisher Scientific, Waltham, Massachusetts), and dried before imaging. For Masson trichrome staining, tissues were hydrated in deionized water, and Bouin solution at $56^{\circ} \mathrm{C}$ was used as the mordant. Sections were stained in working Weigert iron hematoxylin solution and then Biebrich scarlet-acid fuchsin solution. Sections were sequentially incubated in three solutions, including phosphotungstic/phosphomolybdic acid, aniline blue, and $1 \%$ acetic acid, and then mounted. 


\section{Statistical Analyses}

Statistical analyses were performed with the use of GraphPad Prism version 6.02 (GraphPad Software, San Diego, California). Tumor necrosis is reported as the average percent necrosis for all tumors on H\&E-stained histologic sections. Student $t$ test was used to assess the statistical significance of differences in mean values. Fischer exact test was used to compare mortality after the procedure in the lobar versus segmental transarterial embolization groups on an intent-to-treat basis.

\section{RESULTS}

\section{Animal Model Characterization}

The animal model was characterized using two cohorts of 10 animals each of which were treated identically with DEN. Rats administered 0.01\% DEN ad libitum for 12 weeks demonstrated an attenuation in weight gain relative to that reported for untreated animals (Fig 1a) (15). Linear regression analysis demonstrated a biphasic growth kinetic with rats gaining an average of $4.3 \mathrm{~g} / \mathrm{d} \pm 0.1$ (group 1) and $3.2 \mathrm{~g} / \mathrm{d} \pm 0.2$ (group 2) in the first 44 days of DEN administration and an average of $1.0 \mathrm{~g} / \mathrm{d} \pm 0.1$ (group 1) and $1.3 \mathrm{~g} / \mathrm{d} \pm 0.2$ (group 2) thereafter. A statistically significant difference in mean weights was noted between these two groups at only the final time point with mean weights of $353.8 \mathrm{~g} \pm 5.5$ (group 1) and $336.3 \mathrm{~g} \pm 6.0$ (group 2) reflecting the lability in weight loss observed at these late time points $(0.03, \mathrm{n}=2$ per group). By comparison, untreated male Wistar rats demonstrate a sustained rate of growth of $4.8 \mathrm{~g} / \mathrm{d} \pm 0.04$ in the first 105 days of life (14). After the completion of DEN administration, each rat eventually expired as a result of disease or became severely ill requiring euthanasia. The median survival times from initiation of DEN therapy until expiration or euthanasia were 101 days (group 1) and 105 days (group 2) (Fig $1 b)$.

On day 1 after cessation of DEN administration, MR imaging demonstrated the development of tumors in each rat measuring $0.3 \mathrm{~cm}$ in maximum transverse diameter. The average time to the development of a tumor measuring at least $0.5 \mathrm{~cm}$ in minimum transverse diameter on imaging was 88.7 days \pm 4.9 and 90 days \pm 5.3 for the two groups. Necropsy was performed on three animals from each cohort by a veterinary pathologist. At necropsy, each animal demonstrated multiple HCCs (range, 4-12) in the background of severe cirrhosis (Fig 1c-e). Histologic analysis demonstrated an average necrotic fraction of $7 \% \pm 6$. Extrahepatic disease was identified in each animal including pulmonary $(\mathrm{n}=5)$, renal $(\mathrm{n}=2)$, and brain $(\mathrm{n}=1)$ metastases (Fig 1f).

\section{Lobar versus Segmental Transarterial Embolization}

Lobar transarterial embolization of autochthonous HCC bearing rats was technically successful in 9 of 10 rats. After selection of the proper hepatic artery, the 1.5-F catheter was too large to pass beyond the lobar artery (Fig $2 \mathrm{a}-\mathrm{c}$ ). No vasospasm was appreciated on angiography in any of the treated animals. The sole unsuccessful procedure resulted from embolization of the left gastric artery. The average procedure time from skin incision to closure was 132 minutes \pm 10 . Five of the nine successfully treated rats expired within the periprocedural period (within 24 hours of treatment). Necropsy performed on these animals 
demonstrated extensive embolic agents and associated tissue necrosis within the liver ( $n=3)$ and lungs $(\mathrm{n}=2)$ indicating nontarget embolization as the likely cause of death (Fig $2 \mathrm{c}$ ). These animals were likely particularly susceptible to nontarget embolization in the liver given the severity of the DEN-induced cirrhosis and metastatic HCC. Median survival for the four animals surviving the periprocedural period was 12.0 days with histologic analysis demonstrating an average necrotic fraction of $29.5 \% \pm 2.3$. Interval patency of the LCCA was confirmed on ultrasound in each of the surviving four rats at 1 week after treatment (data not shown).

Given the high rate of periprocedural mortality from nontarget embolization using this technique, we developed a more selective approach to mitigate this complication. After selection of the lobar hepatic artery, a 1-F microcatheter was inserted coaxially through the 1.5-F catheter and could be delivered into the segmental hepatic arteries (Fig 3a-c). Selection of the segmental branches of the hepatic arteries was based on celiac arteriography. No vasospasm was appreciated on angiography in any of the treated animals. Superselective embolization of autochthonous HCCs was achieved in 10 of the 13 animals in this cohort. Of the three failures, two resulted from an inability to cannulate beyond the CHA owing to a tortuous celiac artery and sharp angle origin of the CHA (Fig 3d). In one instance, repeated attempts at cannulation of the CHA resulted in dissection (Fig 3e). The third failure was due to the inability to advance the 1-F catheter distal to the selected lobar artery. The average procedure time from skin incision to closure was 127 minutes \pm 24 . Segmental embolization resulted in a decrease in periprocedural mortality $(\mathrm{n}=1, P=.057)$. A single animal surviving the periprocedural period after segmental embolization was euthanized 24 hours following the procedure. Necropsy demonstrated no findings of nontarget embolization in the lungs or liver of this animal (Fig 3d). Segmental embolization was confirmed in these animals based on a significant increase in average necrosis of targeted tumors $(\mathrm{n}=4)$, measuring $49 \%(49.2 \% \pm 15.5$ necrosis $)$, compared with tumor necrosis after lobar embolization $(P=.04)$ (Fig 3e). There was no significant difference in median survival for animals surviving the periprocedural period compared with animals undergoing lobar embolization with a median survival of 12.5 days $(P=.28)$.

\section{DISCUSSION}

These data demonstrate a reproducible technique for achieving superselective transarterial embolization in an autochthonous rat model of HCC. Autochthonous HCCs were induced in each animal in the background of severe cirrhosis. Segmental embolization resulted in a significant increase in tumor necrosis and a reduction in nontarget embolization and associated periprocedural mortality compared with the less selective, lobar embolization technique.

Although the increase in tumor necrosis was anticipated based on the selective delivery of embolic agents to a smaller region, the degree of nontarget embolization in the lobar embolization cohort may have resulted from a combination of several potential causes, including features of the vasculature intrinsic to the model as well as the embolization technique. The vascular resistance of the higher order branch vasculature within the liver in rats is relatively high because of their small size and may have resulted in retrograde flow of

J Vasc Interv Radiol. Author manuscript; available in PMC 2016 May 02. 
embolic agents. Similarly, the microcatheter may have partially obstructed flow within the vessels also contributing to retrograde flow of embolic agents; however, antegrade flow was demonstrated in each of these arteries before embolization. In addition, the arteriovenous shunting seen in larger tumors was likely an important factor. Technical factors including the maximum achievable rate of hand injection of embolic agents also may have contributed to nontarget embolization because more brisk injections would be more likely to overcome the effects of high vascular resistance.

The characterization of the biologic features and capabilities of cancer as well as the underlying molecular mechanisms has led to the elaboration of molecular therapies that may potentiate or be potentiated by ischemia induced by transarterial embolization and transarterial chemoembolization. Many of these innovative strategies focus on the distinctive features of the microenvironment after transarterial embolization and transarterial chemoembolization, including nutrient deprivation leading to an increased glycolytic dependence and an enhanced immune response secondary to antigen presentation $(16,17)$. The continued development and translation of these approaches in particular require biologically relevant animal models of HCC and transarterial embolization and transarterial chemoembolization. Rodent models provide an optimal balance, including low cost and maintenance, the potential for high throughput, physiologic and molecular parallels with humans, and sequenced genomes $(9,18)$. Rat models of HCC in transarterial embolization and transarterial chemoembolization offer versatility with respect to the method of tumor induction.

Various methods of tumor induction have been applied for use in animal models of transarterial embolization and transarterial chemoembolization (19-21). Although orthotopic models offer the benefit of shorter periods of growth latency and precise positioning, they fail to recapitulate the pathophysiology of de novo tumorigenesis and require violation of the liver capsule often leading to peritoneal metastases $(22,23)$. In addition, measurement of the therapeutic response in orthotopic models can be complicated by host immune rejection and spontaneous tumor regression (24). Autochthonous tumors have been shown to recapitulate most nearly the biology of human malignancy with respect to the tumor microenvironment, including tumor vasculature and immunobiology, tumor architecture, and genetic heterogeneity $(23,25,26)$. Autochthonous HCCs may be induced through the administration of chemotoxic agents or through genetic modification. Genetically modified rat models of HCC have been limited but are expected to increase in number with the sequencing of the rat genome. The chemical induction of autochthonous HCCs in rats with oral DEN administration as described in this study is well established $(15,27)$ but has not been previously applied in combination with transarterial embolization and transarterial chemoembolization.

The present study demonstrates the advantages of DEN-induced HCCs for use in experimental studies of transarterial embolization and transarterial chemoembolization. First, DEN administration recapitulated the hepatocellular injury-cirrhosis-malignancy cycle of progression that is seen with human HCC. As a result, the rats develop a similar underlying pathology rendering them susceptible to known complications associated with transarterial embolization and transarterial chemoembolization in humans. Second, the 
appearance of the tumor vascularity on angiography closely paralleled the tumor vascularity seen in human HCCs, including parasitization of adjacent vessels and arteriovenous shunting.

Our data also demonstrate the limitations of this model. First, the time required for tumor development significantly exceeds the time required for surgically implanted or injectable models. Second, in addition to the risk of mortality from excess embolization of a cirrhotic liver, reflux and nontarget embolization may be difficult to detect angiographically in this model, as it would be with other rat models, given the small vessel caliber and volume of embolic agents administered. Third, the induction of background cirrhosis and hepatic dysfunction narrows the therapeutic window compared with models without background liver dysfunction. Finally, the model is limited by the caveat inherent to all rodent models with relation to inference of biologic equivalence and applicability of research findings to human HCC.

Although a biologically relevant animal model is paramount, the ability to mimic, as nearly as possible, the therapeutic approach as applied in humans is of particular importance in studies of transarterial embolization and transarterial chemoembolization before clinical application. Animal models of transarterial embolization and transarterial chemoembolization have been constrained by the intricacies of performing transarterial embolization in small animals. As a result, larger animal models have been favored, and transplantation of VX2 carcinosarcoma into rabbit livers has become the most widely used tumor model for studies of transarterial embolization or transarterial chemoembolization, or both, before clinical application (28); however, the VX2 model does not recapitulate human hepatocarcinogenesis.

Advances in catheter technology have led to the evolution of rat models of transarterial embolization or transarterial chemoembolization, or both, for HCC including retrograde cannulation of the CHA through the gastroduodenal artery via laparotomy and cannulation of the hepatic artery through the femoral artery or LCCA $(15,29-31)$. The present study refines previous approaches to establish a technique that recapitulates the clinical approach of superselective, segmental transarterial embolization, which is believed to preserve better the functional hepatic parenchyma compared with lobar embolization (32). Improved selectivity in transarterial embolization or transarterial chemoembolization, or both, is known to confer additional clinical benefits, including augmented necrosis and chemotherapeutic dosing. The continued patency of the LCCA after the procedure suggests the capability for repeat embolization as is commonly performed in the clinical setting. By mimicking the clinical application of transarterial embolization or transarterial chemoembolization, or both, the superselective technique enables the potential for direct comparisons of different transarterial embolization or transarterial chemoembolization agents, or both, in the same animal and comparisons of treated and untreated (control) tumors in the same animal. In this context, superselective transarterial embolization or transarterial chemoembolization, or both, in a rat model may improve throughput and the consistency of the acquired data. 
Despite these benefits, this technique has inherent limitations. First, the complexity of performing transarterial embolization or transarterial chemoembolization, or both, results in a steep technical learning curve and is time-consuming. Although additional experience will undoubtedly lead to shorter procedure times, the described embolizations required $>2$ hours per animal on average. Second, the technique is most efficient with an assistant to facilitate cannulation of the LCCA after the arteriotomy, although the remaining steps can be accomplished routinely by a single operator.

The present study has several limitations. The study employed a nonrandomized design because the segmental embolization technique was developed in response to the high rate of periprocedural mortality after lobar embolization. As a result, the operators had accrued greater experience in applying the embolization technique to the segmental embolization cohort, which may have influenced the relative periprocedural mortality rates. The tumor size, number, and distribution varied, limiting the inferences that may be drawn by comparisons between degrees of necrosis resulting from segmental versus lobar embolization. In addition, all procedures were performed by the same operator and assistant limiting inferences regarding the adaptability of this technique. Finally, biochemical data, including serum chemistries, were not acquired as part of this study and may have played a role in the observed differences in periprocedural mortality.

In conclusion, this study demonstrates a reproducible technique for segmental embolization in a translational rat model of HCC. Segmental hepatic transarterial embolization resulted in decreased periprocedural mortality relative to lobar hepatic transarterial embolization. The development of a method that mimics clinical transarterial embolization in a clinically relevant model of HCC could help facilitate the in vivo study of transarterial embolization and development of novel therapies to treat HCC.

\section{Acknowledgments}

Grants were received from the SIR Foundation, the National Center for Research Resources Grant UL1-RR024134, which is now at the National Center for Advancing Translational Sciences, Grant UL1TR00-0003, from the Radiological Society of North America, and from T32 Training Grant, during the conduct of the study. The authors thank Hongwei Yu and the Histology Core at the Abramson Family Cancer Research Institute and Marion Knaus and the Small Animal Imaging Facility at the University of Pennsylvania.

\section{ABBREVIATIONS}

$\begin{array}{ll}\text { CHA } & \text { common hepatic artery } \\ \text { DEN } & \text { diethylnitrosamine } \\ \text { H\&E } & \text { hematoxylin-eosin } \\ \text { HCC } & \text { hepatocellular carcinoma } \\ \text { LCCA } & \text { left common carotid artery }\end{array}$

\section{References}

1. Pang RW, Poon RT. From molecular biology to targeted therapies for hepatocellular carcinoma: the future is now. Oncology. 2007; 72(Suppl 1):30-44. [PubMed: 18087180] 
2. Miamen AG, Dong H, Roberts LR. Immunotherapeutic approaches to hepatocellular carcinoma treatment. Liver Cancer. 2012; 1:226-237. [PubMed: 24159587]

3. Arzumanyan A, Reis HM, Feitelson MA. Pathogenic mechanisms in HBV-and HCV-associated hepatocellular carcinoma. Nat Rev Cancer. 2013; 13:123-135. [PubMed: 23344543]

4. Forner A, Llovet JM, Bruix J. Hepatocellular carcinoma. Lancet. 2012; 379:1245-1255. [PubMed: 22353262]

5. Marelli L, Stigliano R, Triantos C, et al. Transarterial therapy for hepatocellular carcinoma: which technique is more effective? A systematic review of cohort and randomized studies. Cardiovasc Intervent Radiol. 2007; 30:6-25. [PubMed: 17103105]

6. Golfieri R, Cappelli A, Cucchetti A, et al. Efficacy of selective transarterial chemoembolization in inducing tumor necrosis in small $(<5 \mathrm{~cm})$ hepatocellular carcinomas. Hepatology. 2011; 53:1580 1589. [PubMed: 21351114]

7. Matsui O. Imaging of multistep human hepatocarcinogenesis by CT during intra-arterial contrast injection. Intervirology. 2004; 47:271-276. [PubMed: 15383735]

8. Falk P. Differences in vascular pattern between the spontaneous and the transplanted $\mathrm{C} 3 \mathrm{H}$ mouse mammary carcinoma. Eur J Cancer Clin Oncol. 1982; 18:155-165. [PubMed: 7201391]

9. De Minicis S, Kisseleva T, Francis H, et al. Liver carcinogenesis: rodent models of hepatocarcinoma and cholangiocarcinoma. Dig Liver Dis. 2013; 45:450-459. [PubMed: 23177172]

10. Li X, Zhou X, Guan Y, Wang YXJ, Scutt D, Gong QY. N-nitrosodiethylamine-induced pig liver hepatocellular carcinoma model: radiological and histopathological studies. Cardiovasc Intervent Radiol. 2006; 29:420-428. [PubMed: 16502159]

11. Burke CT, Cullen JM, State A, et al. Development of an animal model for radiofrequency ablation of primary, virally induced hepatocellular carcinoma in the woodchuck. J Vasc Interv Radiol. 2011; 22(1613-1618):e1. [PubMed: 21959057]

12. Martins PN, Neuhaus P. Surgical anatomy of the liver, hepatic vasculature and bile ducts in the rat. Liver Int. 2007; 27:384-392. [PubMed: 17355461]

13. Ha WS, Kim CK, Song SH, Kang CB. Study on mechanism of multistep hepatotumorigenesis in rat: development of hepatotumorigenesis. J Vet Sci. 2001; 2:53-58. [PubMed: 14614295]

14. Charles River Laboratories. Wistar rat data sheets. 2014. Available at: http://www.criver.com/ products-services/basic-research/find-a-model/wistar-rat. Accessed July 5, 2014

15. Li X, Wang Y-XJ, Zhou X, Guan Y, Tang C. Catheterization of the hepatic artery via the left common carotid artery in rats. Cardiovasc Intervent Radiol. 2006; 29:1073-1076. [PubMed: 16670846]

16. Nakamoto Y, Mizukoshi E, Tsuji H, et al. Combined therapy of trans-catheter hepatic arterial embolization with intratumoral dendritic cell infusion for hepatocellular carcinoma: clinical safety. Clin Exp Immunol. 2007; 147:296-305. [PubMed: 17223971]

17. Ko YH, Pedersen PL, Geschwind JF. Glucose catabolism in the rabbit VX2 tumor model for liver cancer: characterization and targeting hexokinase. Cancer Lett. 2001; 173:83-91. [PubMed: 11578813]

18. Frese KK, Tuveson DA. Maximizing mouse cancer models. Nat Rev Cancer. 2007; 7:645-658. [PubMed: 17687385]

19. Choi JW, Kim H, Kim HC, et al. Blood oxygen level-dependent MRI for evaluation of early response of liver tumors to chemoembolization: an animal study. Anticancer Res. 2013; 33:18871892. [PubMed: 23645735]

20. Thompson SM, Callstrom MR, Knudsen B, et al. Development and preliminary testing of a translational model of hepatocellular carcinoma for MR imaging and interventional oncologic investigations. J Vasc Interv Radiol. 2012; 23:385-395. [PubMed: 22265247]

21. Ju S, McLennan G, Bennett SL, et al. Technical aspects of imaging and transfemoral arterial treatment of N1-S1 tumors in rats: an appropriate model to test the biology and therapeutic response to transarterial treatments of liver cancers. J Vasc Interv Radiol. 2009; 20:410-414. [PubMed: 19167243]

22. Yang R, Rescorla FJ, Reilly CR, et al. A reproducible rat liver cancer model for experimental therapy: introducing a technique of intrahepatic tumor implantation. J Surg Res. 1992; 52:193198. [PubMed: 1538593] 
23. Sikder H, Huso DL, Zhang H, et al. Disruption of Id1 reveals major differences in angiogenesis between transplanted and autochthonous tumors. Cancer Cell. 2003; 4:291-299. [PubMed: 14585356]

24. Buijs M, Geschwind J-FH, Syed LH, et al. Spontaneous tumor regression in a syngeneic rat model of liver cancer: implications for survival studies. J Vasc Interv Radiol. 2012; 23:1685-1691. [PubMed: 23177115]

25. Sharpless NE, Depinho RA. The mighty mouse: genetically engineered mouse models in cancer drug development. Nat Rev Drug Discov. 2006; 5:741-754. [PubMed: 16915232]

26. Becher OJ, Holland EC. Genetically engineered models have advantages over xenografts for preclinical studies. Cancer Res. 2006; 66:3355-3358. discussion 3358-3359. [PubMed: 16585152]

27. Rajewsky MF, Dauber W, Frankenberg H. Liver carcinogenesis by diethylnitrosamine in the rat. Science. 1966; 152:83-85. [PubMed: 5910014]

28. Geschwind JH, Artemov D, Abraham S, et al. Chemoembolization of liver tumor in a rabbit model: assessment of tumor cell death with diffusion-weighted MR imaging and histologic analysis. J Vasc Interv Radiol. 2000; 11:1245-1255. [PubMed: 11099235]

29. Kamphorst EJ, Bödeker H, Koroma S, Linnemann U, Berger MR. New technique for superselective arterial (chemo-) embolization of the rat liver. Lab Anim Sci. 1999; 49:216-219. [PubMed: 10331554]

30. Oberstein PE, Saif MW. Novel agents in the treatment of unresectable neuroendocrine tumors. Highlights from the "2011 ASCO Annual Meeting." Chicago, IL, USA; June 3-7, 2011. JOP. 2011; 12:358-361. [PubMed: 21737896]

31. Cho HR, Choi JW, Kim H-C, et al. Sprague-Dawley rats bearing McA-RH7777 cells for study of hepatoma and transarterial chemoembolization. Anticancer Res. 2013; 33:223-230. [PubMed: 23267149]

32. Pleguezuelo M, Marelli L, Misseri M, et al. TACE versus TAE as therapy for hepatocellular carcinoma. Expert Rev Anticancer Ther. 2008; 8:1623-1641. [PubMed: 18925854] 

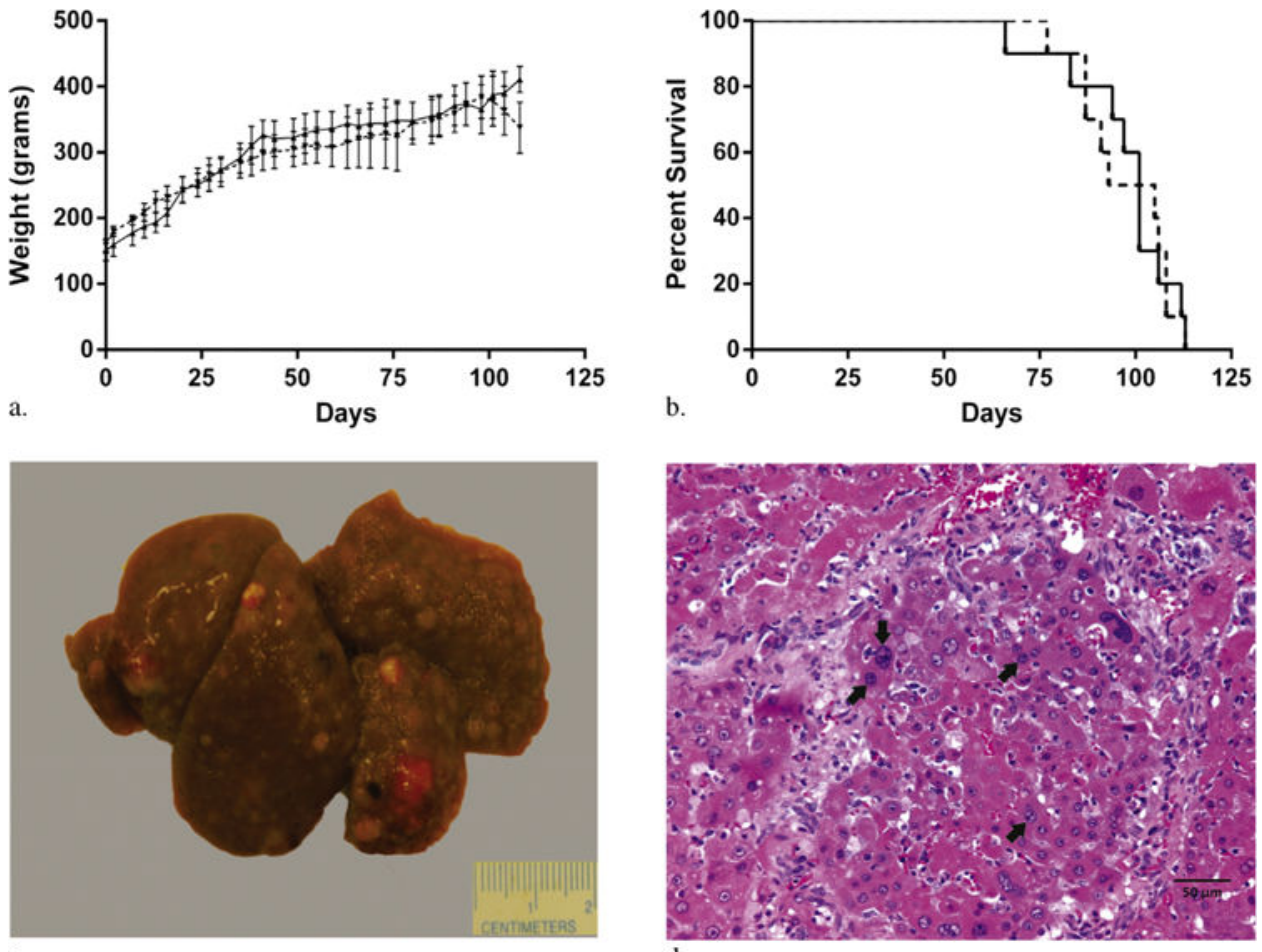

c.
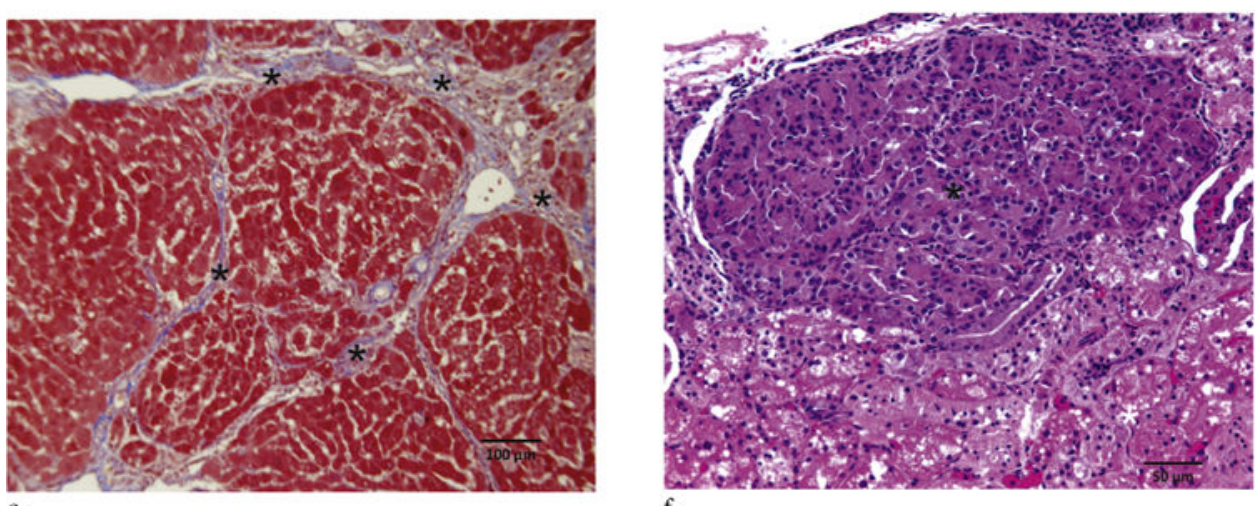

Figure 1.

(a) Plots of weight gain after DEN administration (group 1, - ; group 2--). (b) KaplanMeier curves after DEN administration (group 1, _- group $2--$ ). (c) Representative gross liver specimen 3 weeks after cessation of DEN administration demonstrating a nodular liver with numerous HCCs. (d) HCCs were confirmed on histology with H\&E demonstrating nuclear atypia (arrows). (e) Trichrome staining demonstrated hepatic fibrosis consistent with cirrhosis (asterisks). (f) H\&E staining of renal metastasis (black asterisk) with adjacent normal renal parenchyma (white asterisk) from DEN-treated rat. 

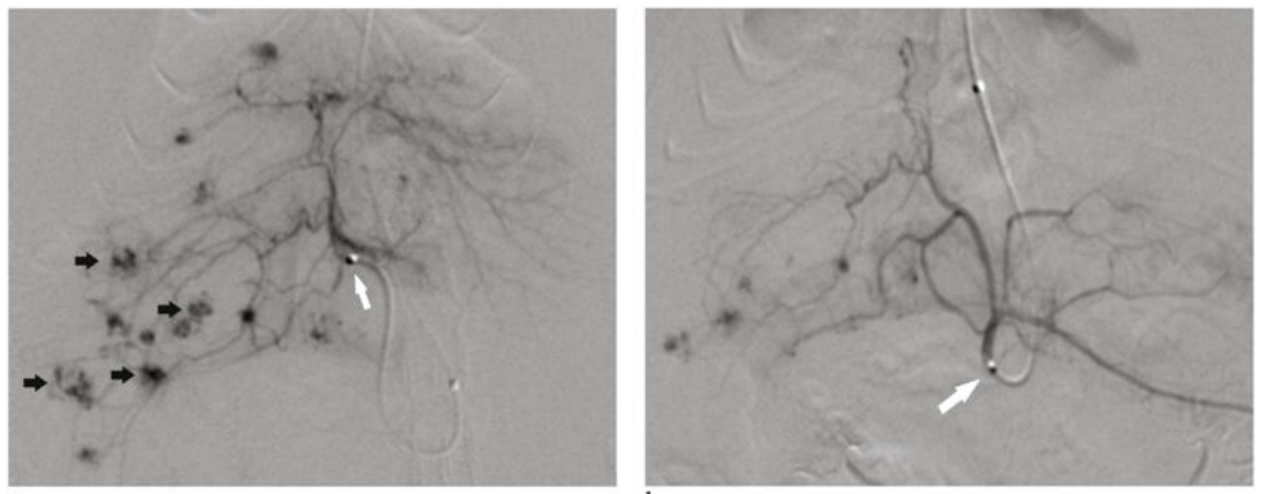

a.

b.

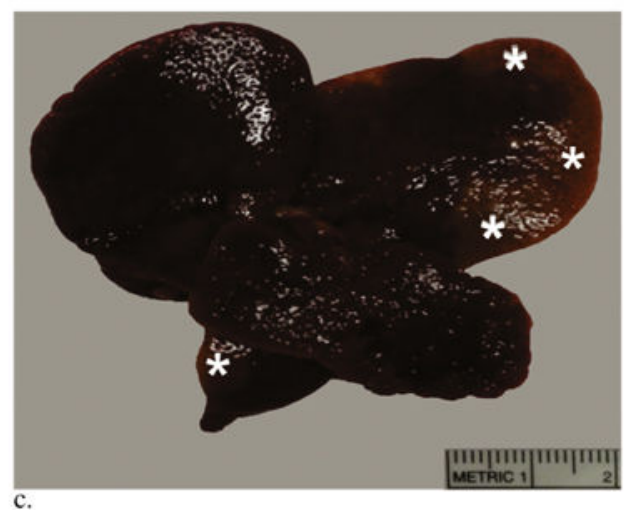

Figure 2.

(a) Representative arteriogram obtained before embolization demonstrating the catheter tip within the right hepatic artery (white arrow) and multiple foci of tumor staining (black arrows). (b) Representative pull-back arteriogram obtained after embolization demonstrating the catheter tip within the celiac artery (arrow) with vascular pruning and exclusion of tumors within the right hepatic lobe. (c) Representative gross image of explanted liver 24 hours after lobar embolization demonstrating regions of peripheral pallor (asterisks) indicating parenchymal ischemia, which suggests nontarget embolization. 


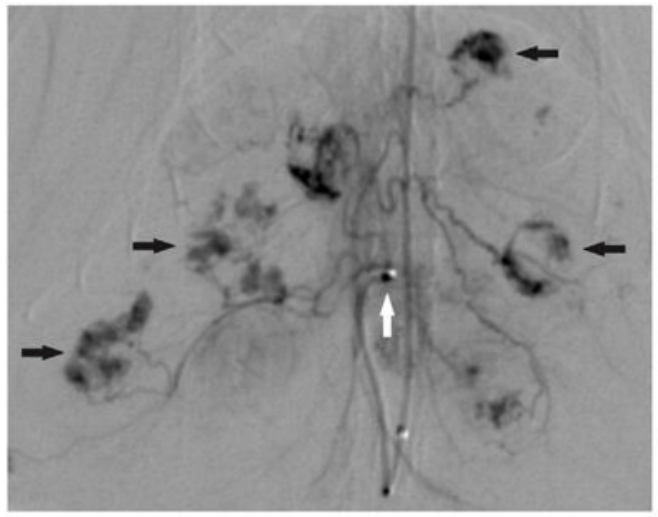

a.

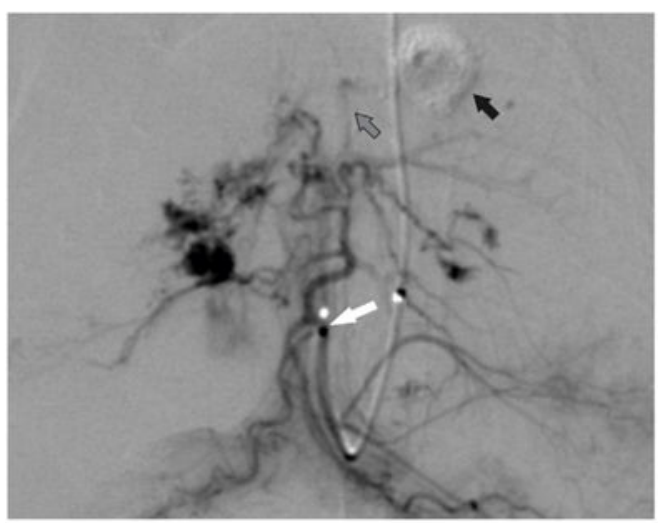

c.

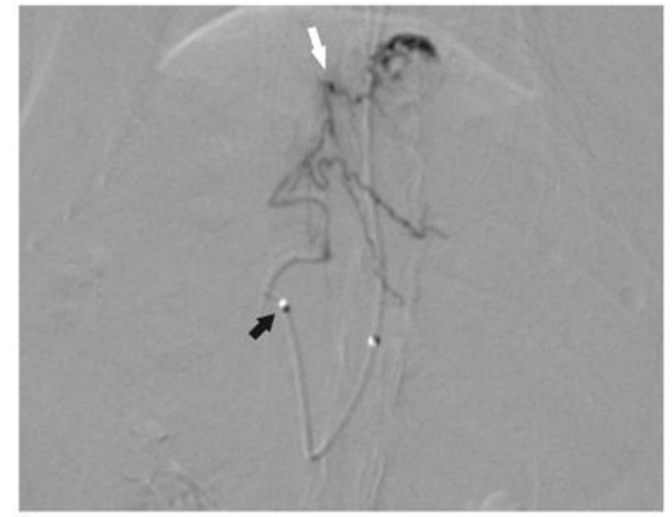

b.

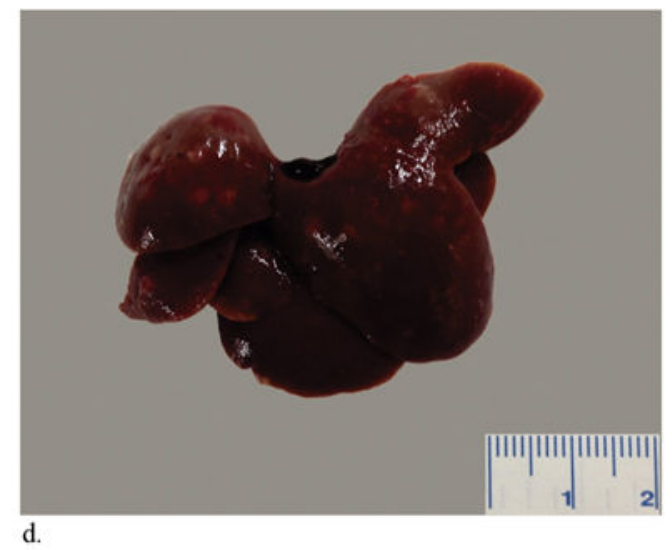

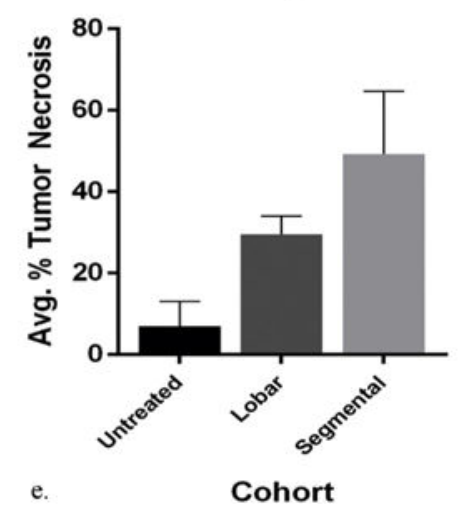

Figure 3.

(a) Representative arteriogram demonstrating the tip of the catheter within the proper hepatic artery (white arrow) and tumor staining (black arrows) within the liver. (b) Representative arteriogram obtained before embolization demonstrating catheter tip within the proper hepatic artery (black arrow) and the microcatheter tip within a second-order branch of the left hepatic artery (white arrow). (c) Representative pull-back arteriogram obtained after embolization demonstrating the catheter tip within the proper hepatic artery (white arrow) with superselective vascular pruning and exclusion of the tumor feeding vessel (gray arrow) and the distal tumor within the left hepatic lobe (black arrow). (d)

Representative explanted liver 24 hours after superselective embolization without findings of nontarget embolization. (e) Bar graph of average percent tumor necrosis in untreated rats 
and rats treated with lobar and segmental embolization demonstrating an expected significant increase in average tumor necrosis for rats treated with segmental embolization relative to lobar embolization $(P=.0029)$. 


\section{Table}

\section{Experimental Cohorts}

\begin{tabular}{lcc}
\hline Cohort & Animals per Cohort & Number of Groups \\
Tumor model characterization & 10 & 2 \\
Transarterial embolization development & 10 & 1 \\
Selective transarterial embolization & 10 & 1 \\
Superselective transarterial embolization & 13 & 1 \\
\hline
\end{tabular}

Note-There were 53 animals treated with diethylnitrosamine. In the first cohort, two groups of 10 animals each were used to characterize diethylnitrosamine-induced cirrhosis and carcinogenesis and overall survival without transarterial embolization (tumor model characterization). A second cohort of 10 animals was used to develop the applied method of transarterial embolization (transarterial embolization development). A third cohort of 10 animals underwent lobar embolization (lobar transarterial embolization). A fourth cohort of 13 animals underwent segmental embolization (segmental transarterial embolization). 